\title{
On Recognizability of Groups by Bottom Layer
}

\author{
Vladimir Senashov ${ }^{1,2}$, Ivan Parashchuk ${ }^{1}$ \\ ${ }^{1}$ Siberian Federal University, Krasnoyarsk, Russia \\ ${ }^{2}$ Institute of Computational Modeling SB RAS, Krasnoyarsk, Russia
}

Corresponding Author Email: sen1112home@mail.ru; ivan-ia-95@mail.ru

https://doi.org/10.18280/ama_a.571-401

Received: 12 June 2020

Accepted: 3 December 2020

\section{Keywords:}

group, bottom layer, spectrum of a group, recognizability, layer-finite group

\begin{abstract}
The bottom layer of a group is a set of its elements of prime order. A group is called recognizable by bottom layer under additional conditions if it is uniquely restored by bottom layer under these conditions. A group is called almost recognizable by bottom layer under additional conditions, if there are a finite number of pairwise non-isomorphic groups satisfying these conditions, with bottom layer that is the same as that of the group. A group is called unrecognizable by bottom layer under additional conditions if there are an infinite number of pairwise non-isomorphic groups satisfying these conditions, with bottom layer that is the same as that of the group. In the paper we consider examples of groups recognized by bottom layer, by spectrum and, simultaneously, by spectrum and by bottom layer. We have also proved some results of recognizability of groups by bottom layer.
\end{abstract}

\section{INTRODUCTION}

The article relates to the description of layer-finite groups, that is, groups in which the set of elements of each order is finite. The applications of such groups are described in article of Senashov "Applications of layer-finite groups" [1]. Now we draw conclusions about the structure of the layer-finite group based on the properties of its bottom layer, that is, set of elements of a prime order. In the future, it is planned to extend these results to a class of almost layer-finite groups using the article by Senashov "Structure of almost layer-finite groups" [2].

The layer-finite groups in our results are not necessarily infinite. Thus, in Theorem 1, a finite simple group $U_{4}(5)$ is recognized. Simple finite groups find applications in studying of symmetric physical systems. Such systems include, in particular, molecules and crystals with symmetry.

Definition. Bottom layer of a group is a set of its elements of prime order.

Definition. A group is called recognizable by bottom layer under additional conditions if it is uniquely restored by bottom layer under these conditions.

Definition. A group is called almost recognizable by bottom layer under additional conditions, if there are a finite number of pairwise non-isomorphic groups satisfying these conditions, with bottom layer that is the same as that of the group.

Definition. A group is called unrecognizable by bottom layer under additional conditions if there are an infinite number of pairwise non-isomorphic groups satisfying these conditions, with bottom layer that is the same as that of the group.

The question of restoring of a group by the bottom layer was considered in [3].

Definition. The spectrum of a finite group is a set of orders of its elements.
Definition. A finite group $G$ is called recognizable by spectrum if any finite group where spectrum coincides with the spectrum of $G$ is isomorphic to $G$.

Definition. A group is said to be almost recognizable by spectrum if there are a finite number of pairwise nonisomorphic groups with the same spectrum as that of the group.

Definition. A group is called unrecognizable by spectrum, if there is an infinite number of pairwise non-isomorphic groups with the same spectrum as that of the group.

Results of recognizability of groups by spectrum can be found in the works of Vasiliev, Mazurov, Staroletov, Zavarnitsin and others.

The paper considers the examples of groups recognized by bottom layer, by spectrum and, simultaneously, by spectrum and bottom layer. Examples of group recognizability are shown.

We have proved several results on recognizability of a group by bottom layer.

\section{EXAMPLES AND THEOREMS}

If bottom layer of a group consists of elements of order $p$ and the number of its components is additionally given, then Kostrikin in 1959 proved the finiteness of the number of finite groups with such conditions [4]. If the set of orders of elements of a bottom layer of an infinite group is small in terms of the number of its constituent numbers, but not in their size, then such examples of groups are quite rare. According to Merzlyakov's metaphoric expression, they are comparable with the «samples of the lunar soil». Such examples include Olshansky's monsters [5]. A series of Olshansky groups for sufficiently large primes $p>10^{10}$ can only be included in the number of the infinite $p$-groups that coincide with its bottom layer in addition to the direct products of cyclic groups of order $p$. 
Let us give examples describing the properties of groups with conditions for bottom layer. Gupta and Mazurov proved that for group $G$ that without unite coincides with its bottom layer consisting of elements of orders 3,5 , one of the statements is true: 1) $G=F T$, where $F$ is a 5-subgroup normal nilpotent of class at most two and $|T|=3$; 2) $G$ contains a normal 3-subgroups $T$ nilpotent of class at most three such that $G / T$ is a 5-group [6]. In the same paper it was shown that a group without unite that coincides with its bottom layer consisting of elements of orders 2, 5, either contains an elementary Abelian 5-subgroup of index 2, or an elementary Abelian normal Sylow 2-subgroup.

By bottom layer of a group, sometimes you can restore a group, sometimes you can say something about the properties of such a group. Among these results, there are those that describe the structure of a group completely by its bottom layer, for example: if bottom layer consists of elements of orders 2, 3, 5 and there are no nontrivial elements of other orders in the group, then Kondratiev and Mazurov proved that this is a group of even substitutions on five elements [7].

Shunkov proved that if bottom layer in an infinite layerfinite group consists of a single element of order 2, then the group is either a quasicyclic or an infinite generalized group of quaternions [8]. We can conclude that such a group is almost recognizable by bottom layer, i.e. there are 2 nonisomorphic groups with the same bottom layer. Many results for groups with a given bottom layer describe only some properties of groups. For example, Mazurov proved that a group with bottom layer consisting of elements $2,3,5$, in which all other non-unit elements have order 4 , is locally finite [9].

Groups $G$ and $H$ are called isospectral if $\omega(G)=\omega(H)$, where $\omega(G)$ is a specrum of group $G$. The number of pairwise non-isomorphic groups isospectral to $G$ is designated by $h(G)$.

In this article we will discuss the relationship between group recognizability by bottom layer and recognizability by spectrum.

Among the results on recognizability by bottom layer are those that describe the structure of the group completely by its bottom layer. For example, if bottom layer of a group consists of elements of order 2 and there are no non-unit elements of other orders in the group, then it is an elementary Abelian 2group. That is, the group under such conditions is recognizable by bottom layer.

An example of an unrecognizable group by spectrum is group $A_{6}$ with a spectrum of 2, 3, 5, 4, 8, 9 (there are infinitely many groups, one of which is $A_{6}$ ) [10]. Group $L_{3}(3)$ with the spectrum $2,3,4,8,9,13,16,27$ is also unrecognizable by spectrum [10].

An example of unrecognizability by bottom layer is given by such groups as $C_{p^{\infty}} \times C_{q}, C_{p^{\infty}} \times C_{q^{2}}, C_{p^{\infty}} \times C_{q^{3}}, \ldots$ where the same bottom layer consists of $p$-1 elements of order $p$ and $q-1$ elements of order $q$

It is proved in [11] that the symmetric groups $S_{n}$ are recognizable by spectrum when $n \in\{2,3,4,5,6,8,10,15,16,18,21,27,33,35,39,45\}$

Grunberg-Kegel graph (or prime graph) $G K(G)$ of group $G$ is defined as follows. The set of vertices of this graph is $\pi(G)$. Different prime numbers $p$ and $q$ from $\pi(G)$ considered as vertices of the graph $G K(G)$, are connected by an edge if and only if $p q \in \omega(G)$.

Until now, all the considered symmetric groups, with the exception of group $S_{9}$, the recognizability of which was proved, had a disconnected prime graph. A symmetric group of degree $n>2$ has a disconnected prime graph only if one of the numbers $n$ or $n-1$ is prime [11].

In 1994, Shi and Brandl proved the recognizability of an infinite series of simple linear groups $L_{2}(q), q \neq 9[12,13]$.

Note that the recognizability of $S_{n}$ groups for $n=7,9,11$, $12,13,14$ and unrecognizability for $n=2,3,4,5,6,8$ was established in [14-17]. The recognizability of group $S_{p}$ was proved by Zavarnitsine [18], where $p$ is a prime number greater than 17. In addition, strong restrictions on groups with the same spectrum as those of group $S_{p+1}$ were also obtained. It was proved in [19] that if group $G$ with the same spectrum as a symmetric group of degree $n$ has a composition factor isomorphic to $A_{n}$, then $G \cong S_{n}$.

Consider some examples.

Let $G$ be a finite group and $\omega(G)=\omega\left(S_{6}(2)\right)$. Then $G$ is isomorphic to $S_{6}(2)$ or $O_{8}^{+}(2)$. In particular, the group $S_{6}(2)$ is almost recognizable by spectrum [16].

Let $S=S_{4}(q)$ be a finite simple symplectic group of dimension 4 over a field of order $q$. If $q==3^{2 n+1}>3$, then $S$ is recognizable by spectrum. In all other cases, $h(S)=\infty$ [20].

Denote by $s=s(H)$ the number of connected components of graph $G K(H)$, and by $\pi_{i}=\pi_{i}(H)$ the $i$-th component of the connection, $i=1, \ldots, s$. If the order of the group $H$ is even, set $2 \in \pi_{1}$. Let $\mu_{i}=\mu_{i}(H)$ (respectively, $\left.\omega_{i}=\omega_{i}(H)\right)$ denote the set consisting of $n \in \mu_{i}(H)(n \in \omega(H))$ such that each prime divisor of $n$ belongs to $\pi_{i}$. Proofs in ref. [20] are given in these terms. The case of even $q$ was considered by Mazurov et al. [21].

Let $L$ be a finite simple exceptional group of Lie type, and $L \neq{ }^{3} D_{4}$ (2). Then any finite group isospectral to $L$ is isomorphic to a finite group $G$, such that $L \leq G \leq$ Aut $L$. In particular, $L$ is almost recognizable by spectrum [22].

As shown by Mazurov [23], the ${ }^{3} D_{4}(2)$ group is indeed an exception: it is unrecognizable by spectrum and quasirecognizable by spectrum at the same time. In fact, this will follow from a series of known results and the quasirecognizability by spectrum of the group $E_{7}(q)$ with $q>3$.

They have another bottom layer, and $G$ and $L$ are unrecognizable by spectrum, but recognizable by bottom layer, i.e. for any $G$ that does not differ from $L$ by spectrum, it differs from $L$ by bottom layer.

A finite simple group $L$ is called quasi-recognizable by spectrum if for any finite group $G$, it follows from the equality $\omega(L)=\omega(G)$ that $G$ contains the only non-Abelian composition factor and it is isomorphic to $L$.

We formulate several theorems for almost recognizability of groups by spectrum.

Let $G$ be a finite simple group $L_{6}(3)$ and $H$ be a finite group with the property $\omega(H)=\omega(G)$.

Then $H \cong G$ or $H \cong G(\gamma)$, where $\gamma$ is a graph automorphism of group $G$ of order 2. In particular, $h(G)=2$ [10].

Let $G$ be a finite group and $\omega(G)=\omega\left(S_{6}(2)\right)$. Then $G$ is isomorphic to $S_{6}(2)$ or $O_{8}^{+}(2)$. In particular, $h(\omega(G))=2$ [14].

Let $G$ be a finite group and $\omega(G)=\omega\left(L_{3}(5)\right)$. Then $G$ is isomorphic to $L_{3}(5)$ or Aut $L_{3}(5)$. In particular, $h(\omega(G))=2$ [23].

The following groups are almost recognizable by spectrum: $O_{8}^{+}(2)[16,24], O_{7}^{+}(3)[24], O_{8}^{+}(3)[24], L_{3}(9)[25,26]$.

We present the information about recognizability by spectrum of simple non-Abelian groups, the orders of elements of which do not exceed 13.

First, we list the groups recognized by spectrum [10], where the orders of elements do not exceed 13 (Table 1). The first 
column of Table 1 contains the designations of the groups recognized by the spectrum. The second column defines the orders of these groups, i.e. the number of elements in the group:

Table 1. Spectrum recognizable groups which orders of elements do not exceed 13

\begin{tabular}{|c|c|}
\hline Group $G$ & Order $G$ \\
\hline$A_{5}$ & $2 * 3 * 5$ \\
\hline$L_{2}(7)$ & $2^{3 * 3 * 7}$ \\
\hline$L_{2}(8)$ & $2^{3 *} * 3^{2 * 7}$ \\
\hline$L_{2}(11)$ & $2 * 3 * 5 * 11$ \\
\hline$L_{2}(13)$ & $2^{3 * 3 * 7 * 13}$ \\
\hline$A_{7}$ & $2^{3 *} 3^{2 * 5 * 7}$ \\
\hline$L_{2}(25)$ & $2^{3 * 3} 3 * 5^{2 *} 13$ \\
\hline$M_{11}$ & $2^{3 *} 3^{2 * 5 * 11}$ \\
\hline$L_{2}(27)$ & $2^{2 * 3^{3 *} * 7 * 13}$ \\
\hline$A_{8}$ & $2^{6 * 3} 3^{2 * 5 * 7}$ \\
\hline$L_{3}(4)$ & $2^{6 * 3} 3^{2 * 5 * 7}$ \\
\hline$S z(8)$ & $2^{6 * 5 * 7 * 13}$ \\
\hline$L_{2}(49)$ & $2^{4 * 3} * 5^{2 * 7^{2}}$ \\
\hline$U_{3}(4)$ & $2^{6 * 3} 35^{2 *} * 13$ \\
\hline$M_{12}$ & $2^{6 * 3} 3^{3 * 5 * 11}$ \\
\hline$A 9$ & $2^{6 * 3} 3^{4 * 5 * 7}$ \\
\hline$L_{2}(64)$ & $2^{6} * 3^{2 * 5 * 7 * 13}$ \\
\hline$M_{22}$ & $2^{7 * 32 * 5 * 7 * 11}$ \\
\hline$U_{4}(3)$ & $2^{7 * 3^{6} * 5 * 7}$ \\
\hline$G_{2}(3)$ & $2^{6 * 3} 3^{6 * 7 * 13}$ \\
\hline $\mathrm{L}_{4}(3)$ & $2^{7 * 36 * 5 * 13}$ \\
\hline${ }^{2} F_{4}(2)$ & $2^{11 * 3^{3} * 5^{2} * 13}$ \\
\hline$A_{11}$ & $2^{7 * 3} 3^{4 * 5^{2 * 7} * 11}$ \\
\hline$H S$ & $2^{9 *} 3^{2 *} * 5^{3 * 7 * 11}$ \\
\hline${ }^{3} D_{4}(2)$ & $2^{12 *} 3^{4 *} 7^{2 *} 13$ \\
\hline$A_{12}$ & $2^{9 *} 3^{5 * 5^{2} * 7 * 11}$ \\
\hline$G_{2}(4)$ & $2^{12 * 3^{3} * 5^{2} * 7 * 13}$ \\
\hline$A_{13}$ & $2^{9 *} 3^{5 * 5^{2} * 7 * 11 * 13}$ \\
\hline $\mathrm{U}_{6}(2)$ & $2^{15 * 3^{6} * 5 * 7 * 11}$ \\
\hline$A_{14}$ & $2^{10 *} 3^{5 * 5^{2} * 7^{2} * 11} * 13$ \\
\hline$L_{5}(3)$ & $2^{9 *} 3^{10 * 5^{*} * 7^{2} * 11^{2} * 13}$ \\
\hline Suz & $2^{13 * 3^{7 *} * 5^{2 * 7 *} 11 * 13}$ \\
\hline$A_{15}$ & $2^{10 * 3^{6} * 5^{3} * 7^{2} * 11} * 13$ \\
\hline$A_{16}$ & $2^{14 *} 3^{6 * 5^{3} * 7^{2} * 11 * 13}$ \\
\hline
\end{tabular}

The following Table 2 shows a list of groups almost recognizable by spectrum (the orders of elements of these groups do not exceed 13) [10].

Table 2. Almost recognizable by spectrum groups which orders of elements do not exceed 13

\begin{tabular}{|c|c|c|}
\hline Group $G$ & Order $G$ & $h(G)$ \\
\hline$S_{6}(2)$ & $2^{9 * 3} 3^{4 * 5 * 7}$ & 2 \\
\hline$L_{3}(9)$ & $2^{7 * 36 * 5 * 7 * 13}$ & 2 \\
\hline$O_{7}^{+}(3)$ & $2^{9 * 3^{9} * 5 * 7 * 13}$ & 2 \\
\hline$U_{4}(5)$ & $2^{7 *} 3^{4 *} * 5^{6 *} 7 * 13$ & 2 \\
\hline $\mathrm{L}_{6}(3)$ & $2^{11 *} 3^{15 *} 5^{*} 7 * 11^{2 *} 13^{2}$ & 2 \\
\hline$S_{6}(3)$ & $2^{9 *} 3^{9 * 5} 5^{*} 7^{*} 13$ & 2 \\
\hline$O_{8}^{+}(2)$ & $2^{12 * 3^{5 *}} 5^{2 * 7}$ & 2 \\
\hline$O_{8}^{+}(3)$ & $2^{12 * 3^{12 *} * 5^{2 *} 7^{*} * 13}$ & 2 \\
\hline
\end{tabular}

The groups from Table 2 are unrecognizable by spectrum, but they are all unique for such a spectrum and given bottom layer.

We show this for the example of group $U_{4}(5)$.

Theorem 1. Let $G$ be a finite simple group $U_{4}(5)$ and $H$ afinite group with the property $\omega(H)=\omega(G)$ and bottom layer the same as that of group $U_{4}(5)$. Then $H \cong G$. That is, group $U_{4}(5)$ is unique for such a spectrum and a bottom layer.
Proof. Indeed, let $G$ be a finite simple group $U_{4}(5)$ and $H$ be afinite group with the property $\omega(H)=\omega(G)$. Vasiliev (Proposition 1) shows that apart from group $U_{4}(5)$, there is only one such group $H \cong G(\gamma)$, where $\gamma$ is a field automorphism of order 2 of the group $G$. Groups $U_{4}(5)$ and $H$ have the same spectrum. At the same time, these groups have different bottom layers, which differ, at least, by an element of order 2. Thus, group $U_{4}(5)$ is unique for such a spectrum and bottom layer. The theorem is proved.

The following Table 3 demonstrates a list of groups that are unrecognizable by spectrum with the order of elements not exceeding 13 [10].

Table 3. Groups those are unrecognizable by spectrum which orders of elements do not exceed 13

\begin{tabular}{|c|c|c|}
\hline Group $G$ & Order G & $h(\mathrm{G})$ \\
\hline $\overrightarrow{A_{6}}$ & $2^{3 *} 3^{2 * 5}$ & $\infty$ \\
\hline$L_{3}(3)$ & $2^{4 * 3^{3 *}} 13$ & $\infty$ \\
\hline$U_{3}(3)$ & $2^{5 * 3} 3^{3 * 7}$ & $\infty$ \\
\hline$U_{4}(2)$ & $2^{6 * 3^{4 * 5}}$ & $\infty$ \\
\hline$U_{3}(5)$ & $2^{4 *} 3^{2 *} * 5^{3 * 7}$ & $\infty$ \\
\hline$J_{2}$ & $2^{7 * 3} 3^{3 * 5} * 7$ & $\infty$ \\
\hline$A_{10}$ & $2^{7 *} 3^{4 * 5^{2} * 7}$ & $\infty$ \\
\hline$S_{4}(5)$ & $2^{6 * 3^{2 *} * 5^{4 *}}$ & $\infty$ \\
\hline$U_{5}(2)$ & $2^{10 * 35 * 5 * 11}$ & $\infty$ \\
\hline$S_{4}(7)$ & $2^{8 *} 3^{2 * 5^{2} * 7^{4}}$ & $\infty$ \\
\hline$S_{4}(8)$ & $2^{9 * 3} 3^{5 * 5^{2} * 7 * 11 * 13}$ & $\infty$ \\
\hline
\end{tabular}

A question here is when groups are unrecognizable by spectrum, but at the same time recognizable by spectrum and by bottom layer. In particular this question is for groups of Tables 2 and 3 .

Let us prove several results for the recognizability of a group by bottom layer.

Theorem 2. Let $G$ be a complete group in which $Z(G)$ is layer-finite and $G / Z(G)$ is a periodic group containing for each prime $p$ only a finite number of p-elements. If in the bottom layer of the group $G$ with $p^{n-1}$ elements of order $p, q^{m-1}$ elements of order $q$, then $G$ is the direct product of $n$ quasicyclic p-groups and $m$ quasicyclic q-groups, which means $G$ is recognizable by bottom layer.

Proof. Let group $G$ satisfy the indicated conditions. Since $Z(G)$ is layer-finite and $G / Z(G)$ is a periodic group containing only finitely many $p$-elements for each simple $p$, then by Proposition 2 the group $G$ is layer-finite. Since, by Proposition 3 , each complete subgroup of the layer-finite group $G$ is contained in the center of the group $G$, since the group $G$ is complete, then it is Abelian. By Proposition 4, the complete Abelian group $G$ decomposes into a direct sum of subgroups isomorphic to the additive group of rational numbers or to quasicyclic groups $C_{p^{\infty}}, C_{q^{\infty}}$, perhaps, by different prime numbers. There can be no rational numbers in this decomposition of groups, since $G$ is a layer-finite group and therefore there are no elements of infinite order in it. Since on the lower layer of the group $G$ we can find only elements of order $p$ and elements of order $q$, then quasicyclic groups can be obtained only by primes $p, q$.

Since in the lower layer of the group $G$ we can find $p^{n-1}$ elements of order $p$, elements $q^{m-1}$ of order $q$, then these factors are $n$ and $m$ respectively.

Thus, we obtained that the group $G$ is recognizable by bottom layer. The theorem is proved.

Theorem 3. Let $G$ be a complete group in which there exists a layer-finite subgroup $S$ at the center of $G$ such that $G / S$ is a 
layer-finite group. If in the bottom layer of the group $G p^{n-1}$ is an element of order $p, q^{m-1}$ is an element of order $q$, then $G$ is a direct product of $n$ quasicyclic p-groups and $m$ quasicyclic q-groups, which means $G$ is recognizable by bottom layer.

Proof. Let a group $G$ satisfy the conditions of the theorem. Since there exists a layer-finite subgroup $\mathrm{S}$ at the center of $G$ such that $G / S$ is a layer-finite group, by Proposition 2, the group $G$ is layer-finite. Since, by Proposition 4, each complete subgroup of the layer-finite group $G$ is contained in the center of the group $G$, and the group $G$ is complete, then it is Abelian. By Proposition 4, the complete Abelian group $G$ decomposes into a direct sum of subgroups isomorphic to the additive group of rational numbers or to a quasicyclic groups, perhaps, by different prime numbers. There can be no rational numbers in this decomposition of groups, since $G$ is a layer-finite group and therefore there are no elements of infinite order in it. Since on the lower stratum of the group $G p^{n-1}$ is an element of order $p$ and $q^{m-1}$ is an element of order $q$, then quasicyclic groups can be obtained only by primes $p, q$. Since in the lower stratum of the group $G$ we can find $p^{n-1}$ elements of order $p$ and $q^{m-1}$ elements of order $q$, then there are $n$ and $m$ factors respectively. The theorem is proved.

Theorem 4. Let $G$ be a complete group in which there exists a layer-finite subgroup $S$ at the center of $G$ such that $G / S$ is a layer-finite group. If in the lower layer of a group $G$ is $p_{1}^{m_{1}}-$ 1 element of order $p_{1}, p_{2}^{m_{2}}-1$ element of order $p_{2}, \ldots, p_{n}^{m_{n}}-$ 1 element of order $p_{n}$, then $G$ is the direct product $m_{1}$ of quasicyclic $p_{1}$-groups, $m_{2}$ of quasicyclic $p_{2}$-groups, ..., $m_{n}$ of quasicyclic $p_{n}$-groups, i.e. it is recognizable by bottom layer.

Proof. Let the group G satisfy the conditions of the theorem. Since there exists at the center of $G$ a layer-finite subgroup $S$ such that $G / S$ is a layer-finite group, by Proposition 2, the group $G$ is layer-finite. Since, by Proposition 4, each complete subgroup of the layer-finite group $G$ is contained in the center of the group $G$, since the group $G$ is complete, then it is Abelian. By Proposition 4, the complete Abelian group $G$ decomposes into a direct sum of subgroups isomorphic to the additive group of rational numbers or to quasicyclic groups perhaps, by different prime numbers. There can be no rational numbers in this decomposition of groups, since $G$ is a layerfinite group and therefore there are no elements of infinite order in it. Since on the lower layer of the group $G$ there are only elements of orders $p_{1}, p_{2}, \ldots, p_{n}$, then quasicyclic groups $C_{p_{1}^{\infty}}, C_{p_{2}^{\infty}}, \ldots, C_{p_{n}^{\infty}}$ can be only by prime numbers $p_{1}, p_{2}, \ldots, p_{n}$. Since in the bottom layer of the group $G$ is $p_{1}^{m_{1}}-1$ elements of order $p_{1}, p_{2}^{m_{2}}-1$ elements of order $p_{2}, \ldots, p_{n}^{m_{n}}-$ 1elements of order $p_{n}$, then these factors are $m_{1}, m_{2}, \ldots, m_{n}$, respectively, hence $G$ is recognizable by bottom layer. The theorem is proved.

Proposition 1 (Vasiliev theorem [22]). Let G be a finite simple group $U_{4}(5)$ and $H$ a finite group with the property $\omega(H)$ $=\omega(G)$. Then $H \cong G$ or $H \cong G(\gamma)$, where $\gamma$ is a field automorphism of the group $G$ of order 2. In particular, $h(G)=$ 2 .

Definition. $h(G)$ is the number of pairwise nonisomorphic finite groups that are isospectral to the group $G$.

Proposition 2 (Baer theorem [27]). The following properties are equivalent;

a) $G$ is a layer-finite group;

b) $Z(G)$ is layer-finite and $G / Z(G)$ is a periodic group containing for each prime $p$ only a finite number of $p$-elements;

c) there exists a subgroup $S$ at the center of $G$ such that $S$ and $G / S$ are layer-finite groups.
Proposition 3 (Lemma 3.1 from [28]). Each complete subgroup of a locally normal (in particular, layer-finite) group is contained in the center of the group.

Proposition 4 (Theorem 9.1.6 from [29]). A non-zero complete Abelian group decomposes into a direct sum of subgroups isomorphic to the additive group of rational numbers or to quasicyclic groups perhaps by different prime numbers.

c) for every prime number $p$, there exists at least one Sylow $p$-subgroup in $G$, which is a layer-finite group.

\section{CONCLUSION}

We consider the examples of groups recognized by bottom layer, by spectrum and simultaneously by spectrum and bottom layer. We proved several results on the recognizability of a group by bottom layer.

\section{REFERENCES}

[1] Senashov, V.I. (1989). Characterization of layer-finite groups. Algebra and Logic, 28(6): 449-461.

[2] Senashov, V.I. (2011). Structure of Almost Layer-Finite Groups. AMSE Transactions, Advances in Modeling and Analysis, 48(1): 28-38.

[3] Parashchuk, I.A. (2017). Restoration of the group by bottom layer. Actual problems of aviation and astronautics, 2: 296-297.

[4] Kostrikin, A.I. (1959). The Burnside problem, Izv. of USSR Academy of Sciences. Ser. Math., 23(1): 3-34. http://mi.mathnet.ru/eng/izv/v23/i1/p3

[5] Ol'shanskii, A.Y.E. (1980). An infinite group with subgroups of prime orders. Izvestiya Rossiiskoi Akademii Nauk. Seriya Matematicheskaya, 44(2): 309321. http://mi.mathnet.ru/eng/izv/v44/i2/p309

[6] Gupta, N.D., Mazurov, V.D. (1999). On groups' with small orders of elements. Bulletin of the Australian Mathematical Society, 60(2): $\quad$ 197-205. https://doi.org/10.1017/S0004972700036339

[7] Kondrat'ev, A.S., Mazurov, V.D. (2000). Recognition of alternating groups of prime degree from the orders of their elements. Sibirskii Matematicheskii Zhurnal, 41(2): 359-369. http://mi.mathnet.ru/eng/smj/v41/i2/p359

[8] Shunkov, V.P. (1970). On a class of $P$-groups. Algebra and Logic, 9(4): 291-297.

[9] Mazurov, V.D. (2000). Groups of exponent 60 with prescribed orders of elements. Algebra and Logic, 39(3): 189. https://doi.org/10.1007/BF02681762

[10] Vasil'ev, A.V. (2005). On recognition of all finite nonabelian simple groups with orders having prime divisors at most 13. Siberian Mathematical Journal, 46(2): 246-253. https://doi.org/10.1007/s11202-005-0024-z

[11] Gorshkov, I.B. (2014). Recognizability of symmetric groups by spectrum. Algebra I logika, 53(6): 693-703. https://doi.org/10.1007/s10469-015-9306-0

[12] Shi, W.J. (1987). A characteristic property of $J_{1}$ and $P S L_{2}$ $\left(2^{n}\right)$. Adv. Math, 16: 397-401.

[13] Brandl, R., Shi, W.J. (1994). The characterization of $\operatorname{PSL}(2, q)$ by its element orders, J. Algebra, 163(1): 109114.

[14] Brandl, R., Shi, W.J. (1991). Finite groups whose element orders are consecutive integers. Journal of 
Algebra, 143(2): 388-400.

[15] Praeger, C.E., Shi, W. (1994). A characterization of some alternating and symmetric groups. Communications in Algebra, 22(5): $1507-1530$ https://doi.org/10.1080/00927879408824920

[16] Mazurov, V.D. (1997). Characterizations of finite groups by sets of orders of their elements. Algebra and Logic, 36(1): 23-32. https://doi.org/10.1007/BF02671951

[17] Darafsheh, M.R., Moghaddamfar, A.R. (2000). A characterization of some finite groups by their element orders. In Algebra Colloquium, 7(4): 467-476.

[18] Zavarnitsine, A.V. (2002). Recognition by the set of element orders of symmetric groups of degree $r$ and $r+1$ for prime r. Siberian Mathematical Journal, 43(5): 808811. https://doi.org/10.1023/A:1020142420831

[19] Zavarnitsin, A.V., Mazurov, V.D. (1999). Element orders in coverings of symmetric and alternating groups. Algebra and Logic, 38(3): 159-170. https://doi.org/10.1007/BF02671740

[20] Mazurov, V.D. (2002). Recognition of finite simple groups $S_{4}(q)$ by their element orders. Algebra and Logic, 41(2): 93-110. https://doi.org/10.1023/A:1015356614025

[21] Mazurov, V.D., Xu, M.C., Cao, H.P. (2000). Recognition of finite simple groups $L_{3}\left(2^{\mathrm{m}}\right)$ and $U_{3}\left(2^{\mathrm{m}}\right)$ by their element orders. Algebra and Logic, 39(5): 324-334.
https://doi.org/10.1007/BF02681616

[22] Vasil'ev, A.V., Staroletov, A.M. (2015). Almost recognizability by spectrum of simple exceptional groups of Lie type. Algebra and Logic, 53(6): 433-449. https://doi.org/10.1007/s10469-015-9305-1

[23] Mazurov, V.D. (2013). Unrecognizability of a finite simple group ${ }^{3} D_{4}$ (2) by spectrum. Algebra and logic, 52(5): 400-403.

[24] Shi, W.J. Tang, C.Y. (1997). A characterization of some orthogonal groups. Progr. Nat. Sci., 7(2): 155-162.

[25] Zavarnitsine, A.V. (2004). The weights of irreducible $L_{3}(q)$-modules in the defining characteristic. Siberian Mathematical Journal, 45(2): 261-268. https://doi.org/10.1023/B:SIMJ.0000021282.92576.f8

[26] Zavarnitsine, A.V. (2003). Recognition of the simple groups $L_{3}(q)$ by element orders. Journal of Group Theory, 7(1): 81-97. https://doi.org/10.1515/jgth.2003.044

[27] Baer, R. (1948). Finiteness properties of groups. Duke Mathematical Journal, 15(4): 1021-1032. https://doi.org/10.1215/S0012-7094-48-01591-9

[28] Chernikov, S.N. (1958). On layer-finite groups. Mat. Sb., 45(3): 415-416.

[29] Kargapolov, M.I., Merzlyakov, Y.I. (1982). Fundamentals of group theory. Ed. 3rd, Moscow: Science, 1-288. 